\title{
Immunity of electronic devices against radio-frequency electromagnetic fields
}

\author{
Hana Urbancokova ${ }^{1^{*}}$, Stanislav Kovar ${ }^{1}$, Ondrej Halaska ${ }^{1}$, and Jan Valouch ${ }^{1}$ \\ ${ }^{1}$ Faculty of Applied Informatics, Tomas Bata University in Zlin, nam. T.G.Masaryka 5555, 76001 Zlin, Czech Republic
}

\begin{abstract}
One of the major types of electromagnetic interference, which affect electronic devices in their normal operation, is the interference with radio-frequency electromagnetic fields. This interference is generated by the mainly radio and television transmitters, industrial equipment and other transmitters and receivers in general use for communication. Testing of electromagnetic susceptibility of electronic devices on radiated radio-frequency electromagnetic field is governed by the basic standard IEC 61000-4-3 and the equipment under test are exposed to test electromagnetic fields with an intensity from $1 \mathrm{~V} / \mathrm{m}$ to $30 \mathrm{~V} / \mathrm{m}$, the most often in the frequency range from $80 \mathrm{MHz}$ to $2 \mathrm{GHz}$. The aim of this paper is to explain the issue of electromagnetic susceptibility and to present sample the electromagnetic immunity tests of the basic set of the intrusion and hold-up alarm system against the radio-frequency electromagnetic field according to the relevant electromagnetic compatibility standards.
\end{abstract}

\section{Introduction}

Measurement and testing of electromagnetic compatibility (EMC) of electronic devices are carried out according to standardized methodologies in a precisely defined environment. An ideal environment for testing the electromagnetic susceptibility of electronic devices would be the real environment in which the devices will work. However, such the environment is variable. So because of the reproducibility of the test, a simulated environment is selected according to the instructions that are given in the standards of the individual immunity tests.

Measurement of interference which is transmitted by the radiation is performed in anechoic or semi-anechoic chambers; respectively at the test site in open space OATS - Open Area Test Site. Testing for electromagnetic immunity in the test radiated radiofrequency electromagnetic field should be always carried out in shielded chambers due to the high intensity of the generated field. But in the case of large systems or devices, the measurements must be carried out at the installation site. [1,2]

Electromagnetic susceptibility (EMS) is the ability of a device, equipment or system to function without degradation in the presence of an electromagnetic disturbance. They must work without failures or with a precisely defined allowable influence. EMS deals with the technical measures which increase electromagnetic immunity of receivers. EMS is focused on removing the consequences of interference, without removing their causes.

The electromagnetic susceptibility of the technical system is divided into:
- Internal EMS - it is system immunity against interfering sources contained inside its own system.

- External EMS - it is the resilience of the system against external sources of electromagnetic interference (EMI). [2, 3]

This paper describes the electromagnetic immunity test of the basic set of the intrusion and hold-up alarm system (I\&HAS). This basic set consisted of a control panel with an accumulator stored in a plastic box, the keypad, PIR detector and siren. This set has been tested to the effects of EMI (radiated, radio-frequency, electromagnetic field), and system failures or unintentional behaviour of components of I\&HAS were monitored during the immunity tests. A semi-anechoic chamber was used for EMS tests and the test set was in two basic states (ON state and state of alarm).

\section{Radio-frequency electromagnetic field}

Testing of electromagnetic susceptibility of electronic devices on radiated radio-frequency electromagnetic field is governed by the basic standard IEC 61000-4-3 Electromagnetic compatibility (EMC) Part 4-3: Testing and measurement techniques - Radiated, radiofrequency, electromagnetic field immunity test.

Radio-frequency field is mostly generated by a tiny system transmitter/receiver used by staffs that ensure operation, maintenance and safety in manufacturing. Other systems that generate radio-frequency electromagnetic fields can be, for example, stable radio or television transmitters, vehicles transmitters, and other industry electromagnetic sources. In the last years, a large growth of radio-frequency interference was

\footnotetext{
* Corresponding author: $\underline{\text { urbancokova } @ \text {,fai.utb.cz }}$
} 
detected, especially in the frequency bands from 0.8 $\mathrm{GHz}$ up to $6 \mathrm{GHz}$. [2, 4]

\subsection{Testing levels}

The following table (Table 1.) shows an intensity of the testing field based on testing level according to IEC 61000-4-3. Testing levels are used for general purposes, digital radiotelephones and other devices which emit radio-frequency radiation.

Table 1. Intensity of radio-frequency radiation

\begin{tabular}{|c|c|}
\hline Level & $\begin{array}{c}\text { Intensity of testing field } \\
\mathbf{V} / \mathbf{m}\end{array}$ \\
\hline 1 & 1 \\
\hline 2 & 3 \\
\hline 3 & 10 \\
\hline 4 & 30 \\
\hline $\mathrm{X}$ & Special \\
\hline
\end{tabular}

"X" represents the unlimited testing level and its associated field intensity can acquire whatever value. This testing level can be given in the product standard.

The intensity of the testing field is represents as a value of an unmodulated signal. This signal is modulated by sinus wave $1 \mathrm{kHz}$ with $80 \%$ modulation depth when testing of electromagnetic susceptibility. The real threat when radio-frequency fields radiating is simulated for the equipment under test (EUT) by this modulation. [4]

\subsection{Environmental classes}

The selection of the appropriate level for EUT is made according to environmental class selection based on the followings:

- Class 1: The environment with the low levels of the electromagnetic radiation - typical environment for the local radio stations or television stations that are further than $1 \mathrm{~km}$ each other and low power transmitters/receivers.

- Class 2: The environment with the moderate levels of the electromagnetic radiation - typical for business environment in which the low power transmitters are used (less than $1 \mathrm{~W}$ ) but with closeto-device-usage limitation.

- Class 3: Then environment with the demanding levels of the electromagnetic radiation - typical for the industry environment where are transfer receivers with the higher power than $2 \mathrm{~W}$, which are used in the close field of the device (up to $1 \mathrm{~m}$ ). There is also the environment, where the radio transmitters, industry, scientific or medical devices are used.

- Class 4: Transfer systems transmitter/receiver and significant sources of interference are situated at the distance of less than $1 \mathrm{~m}$ from the EUT.

- Class X: The unlimited level which is defined in a standard for particular product or in a device specification. [4, 5]

\subsection{Frequency range}

Testing of electromagnetic susceptibility on the general purposes is mostly done in frequency range from 80 $\mathrm{MHz}$ up to $2 \mathrm{GHz}$. In case, when testing levels involve safety against radio-frequency radiation from digital radiotelephones and other devices which emit radiofrequency fields, the immunity tests are done in frequency range from $800 \mathrm{MHz}$ up to $960 \mathrm{MHz}$ and 1.4 $\mathrm{GHz}$ up to $6.0 \mathrm{GHz}$.

\section{Workplace for EMS testing}

The basic principle of testing of susceptibility against the radio-frequency electromagnetic field is the use of appropriate antennas for irradiation EUT by specific levels of the electromagnetic field. This testing should be done inside absorption places (in an anechoic chamber or semi-anechoic chamber with additional absorbers).

Workplace for EMS testing consists of technical and other equipment such as the high-frequency signal generator with amplitude modulation sinus wave $1 \mathrm{kHz}$ with $80 \%$ modulation depth. The output of the generator can have a filter at the bottom sluice band for harmonic features suppression of the generated signal.

Other equipment which is needed for susceptibility testing of electronic devices is broadband powerful amplifier (for signal amplification), the transmission directional antenna which emits testing electromagnetic wave (generates electromagnetic fields), for example, biconical (double-cone) antenna, logarithmic-periodic antenna, funnel antenna or antenna with double waveguide or another linear polarized antenna system which is appropriated for frequency requirements. In all inputs and outputs of cables and conducting to the testing chamber must be plugged the electric filters EMI (the filter must not to produce additional resonance on the plugged conducting). The isotropic sensor of the field with appropriate susceptibility and auxiliary device for recording power levels, for checking and for continuous functional evaluating EUT and other equipment for ensuring specific function when testing, are needed.

In the tests of susceptibility to radio-frequency fields, most of the EUTs are placed in an absorption chamber on an $80 \mathrm{~cm}$ high table, or on a floor on a nonconductive pad 5-15 cm high. According to standards preferred distance of the antenna from the EUT is $3 \mathrm{~m}$. If this distance is can not be met, it may be shorter, but not less than $1 \mathrm{~m}$. Also, the distance of the EUT from the walls of the chamber should be greater than $1 \mathrm{~m}$. The EUT should be irradiated by a homogeneous field of known and constant intensity. The area of this field is usually $1.5 \times 1.5 \mathrm{~m}$ in height of $0.8 \mathrm{~m}$ above the floor. In case of testing smaller devices, the area of the field may be smaller, but it must not fall below $0.5 \times 0.5 \mathrm{~m}$.

The test procedure includes four points:

a) Verification of laboratory reference conditions.

b) Verification of the proper operation of the EUT.

c) Performing the test.

d) Evaluation of test results. [4, 5, 6] 


\subsection{Equipment under test}

Fig. 1 shows the basic set of intrusion and hold-up alarm systems, which was tested for resistance against radiofrequency interference. For this type of equipment it is necessary to comply not only the basic standard IEC 61000-4-3, but also the next specialized standard EN 50130-4 Alarm systems - Part 4: Electromagnetic compatibility - Product family standard: Immunity requirements for components of fire, intruder, hold up, CCTV, access control and social alarm systems. [7]

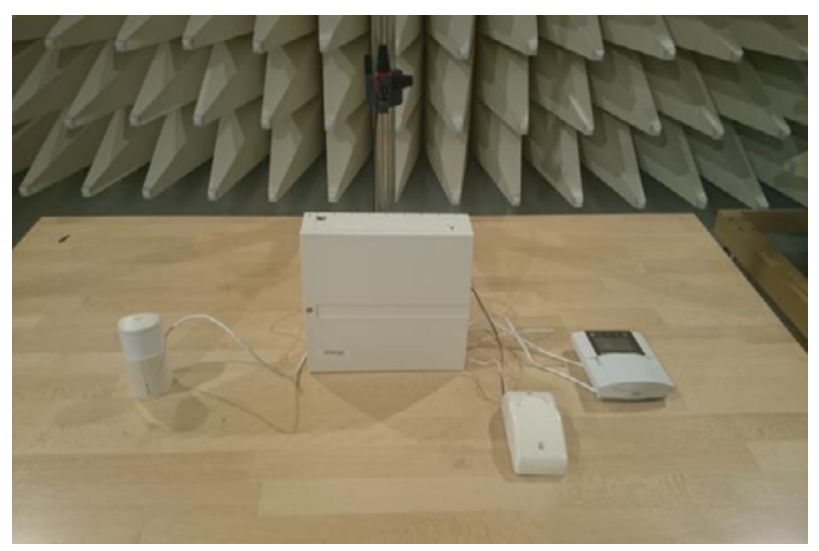

Fig. 1. The basic set of I\&HAS.

The set was powered from the mains $240 \mathrm{~V} / 50 \mathrm{~Hz}$. The control panel, accumulator and mains power module were closed in the plastic box which is usually supplied with the control panel. All components belong to the product lines Oasis. Fig. 1 shows the location of the components of I\&HAS on the table which was placed on the turntable in the semi-anechoic chamber.

The set included the following components:

- Control panel JA 82-K.

- Accumulator 12V, 2.4Ah.

- Mains power module.

- Keypad JA-81E.

- PIR detector JS-20.

- Siren SA-913TM.

\subsection{Measuring equipment}

The measurements of electromagnetic sesceptibility were performed in the semi-anechoic chamber in the EMC laboratory at the Tomas Bata University in Zlin. The semi-anechoic chamber creates a shielded space in which all inappropriate electro-magnetic interferences from the environment that could distort the measurement results are eliminated. [8]

Used semi-anechoic chamber from manufacturer FRANCONIA was equipped with the logarithmicperiodic antenna HL046 (Rohde\&Schwarz) (Fig. 2) which operates in the frequency range from $80 \mathrm{MHz}$ to $1.3 \mathrm{GHz}$. The distance of the antenna from the equipment under test is adjustable according to the requirements of standards.

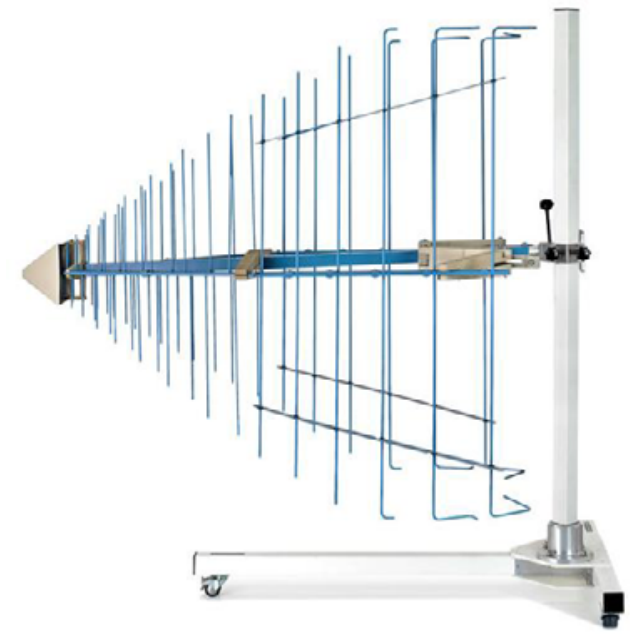

Fig. 2. Logarithmic-periodic antenna HL046.

Another device in a semi-anechoic chamber was electric field probe HI-6015 (ETS-Lindgren) which operates in the frequency range from $100 \mathrm{kHz}$ to $6 \mathrm{GHz}$.

Other measuring equipment were placed outside the semi-anechoic chamber:

- Signal generator - SMB 100A (Rohde\&Schwarz) frequency range from $9 \mathrm{kHz}$ to $6 \mathrm{GHz}$.

- EMI test receiver - ESU (Rohde\&Schwarz) frequency range from $20 \mathrm{~Hz}$ to $8 \mathrm{GHz}$.

- Amplifier No.1 - 150A250 (Amplifier Research) frequency range from $100 \mathrm{kHz}$ to $250 \mathrm{MHz}$.

- Amplifier No.2 - 150W1000 (Amplifier Research) frequency range from $80 \mathrm{MHz}$ to $1 \mathrm{GHz}$.

- Amplifier No.3 - 80S1G4 (Amplifier Research) frequency range from $0.7 \mathrm{GHz}$ to $4.2 \mathrm{GHz}$.

- Switch and control unit - OSP 130 (Rohde\&Schwarz).

- Switch and control unit - OSP 150 (Rohde\&Schwarz).

- EMC measurement software - EMC32 (Rohde\&Schwarz). [9]

\section{The results of selected measurements}

The EUT, on which was measured the electromagnetic susceptibility to radiated electromagnetic fields, was tested in the semi-anechoic chamber in the EMC laboratory at the Tomas Bata University in Zlin. The set of I\&HAS was measured in the mode where the whole set was in the ON state (state of guarding) or when the alarm was induced. The distance of the antenna from the EUT was $2.5 \mathrm{~m}$, in order to ensure the required strength of an electromagnetic field. The testing was performed using a Peak detector which indicates the maximum value of the electromagnetic field for each measured frequency.

The selected measurements from the semi-anechoic chamber are shown in Figures 3, 4, 5 and 6. A harmonic unmodulated signal (which corresponds to the values of field strength) is amplitude modulated to a depth of $80 \%$ by the low-frequency harmonic voltage of $1 \mathrm{kHz}$. The strength of the electric field should be set to a value of $10 \mathrm{~V} / \mathrm{m}$. 
Each test run is shown using two images that are outputs from the EMC32 control and display software. The $\mathrm{x}$-axis shows the frequency in $\mathrm{Hz}$ from $80 \mathrm{MHz}$ to 3 GHz. The y-axis shows either the degree of the radiated electromagnetic field (Imm Level) in $\mathrm{V} / \mathrm{m}$ (volt per meter) or the level of the currently used amplifier (Amp Out Fwd) in W (watt).

According to standard IEC 61000-4-3 the frequency range from $80 \mathrm{MHz}$ to $2 \mathrm{GHz}$ is the most often to used, but we have set the frequency value up to $3 \mathrm{GHz}$, since standard EN 50130-4 recommends testing EMS frequencies up to $2.7 \mathrm{GHz}$.

In the first EMS test, I\&HAS test set was in the ON state (state of guarding). During the test in the prescribed frequency range $(80 \mathrm{MHz}$ to $2 \mathrm{GHz}$ - according to IEC 61000-4-3), the assembly resisted the action of a radiofrequency field of level 3 , which corresponding to the intensity of the field of radiation of $10 \mathrm{~V} / \mathrm{m}$. After exceeding the $1.988 \mathrm{GHz}$ frequency, the status of the EUT has changed, the system detected an error and proclaimed an alarm (the siren was activated).

In Fig. 3 the intensity of the test field at individual frequencies is shown where deviations from the required $10 \mathrm{~V} / \mathrm{m}$ are usually minimal. At $175 \mathrm{MHz}$ and $810 \mathrm{MHz}$ the deviation was greater $(-1.25 \mathrm{~V} / \mathrm{m})$. Fig. 4 shows the power of the amplifiers during the test.

In the next test, I\&HAS test set was in the alarm state (when the alarm was induced and the siren has been activated). During the entire test (from $80 \mathrm{MHz}$ to 3 $\mathrm{GHz}$ ), the status of the EUT has not changed and the system did not report no errors after the test was completed. The intensity of the test field at individual frequencies and the power of the amplifiers during the second test are shown in Fig. 5 and Fig. 6.

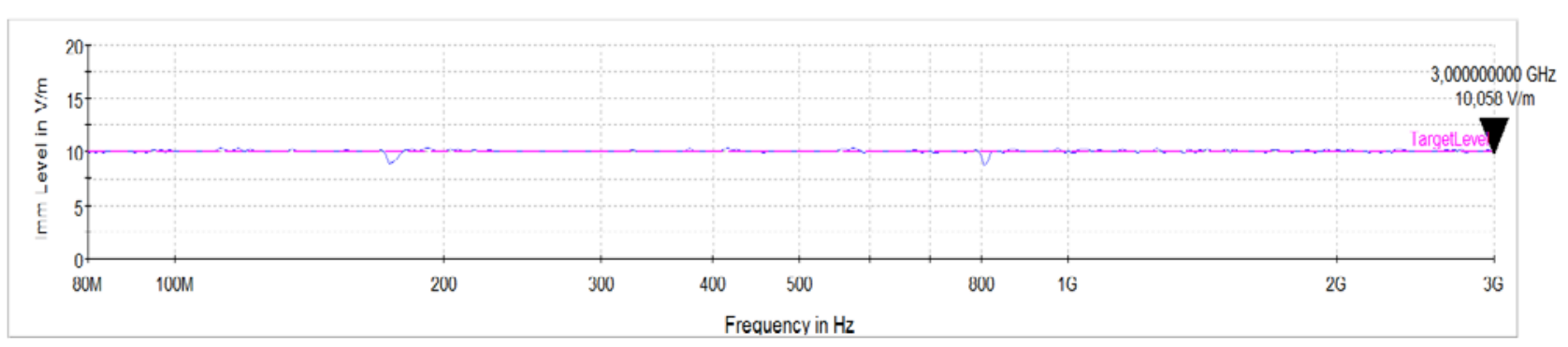

Fig. 3. Test 1 - Immunity level.

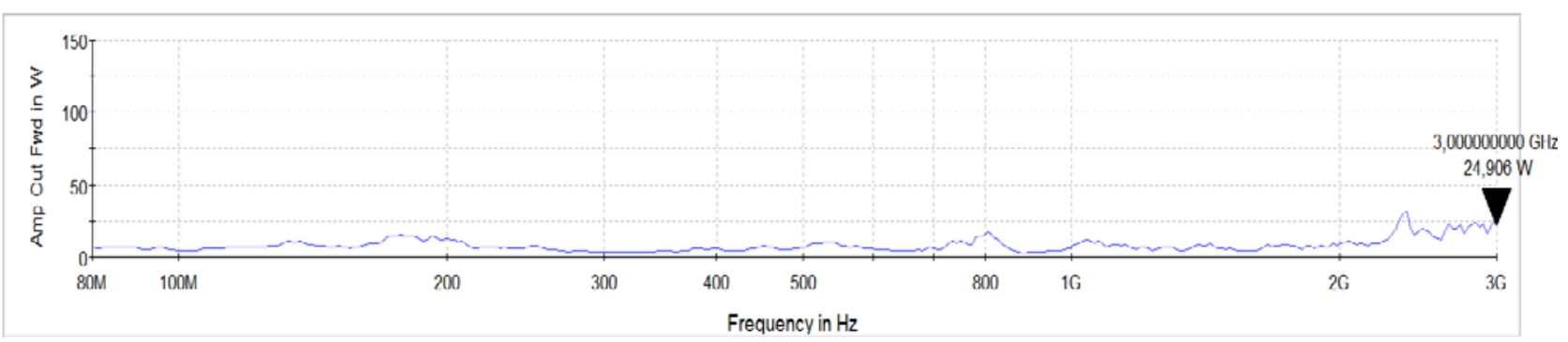

Fig. 4. Test 1 - Amp Out Fwd.

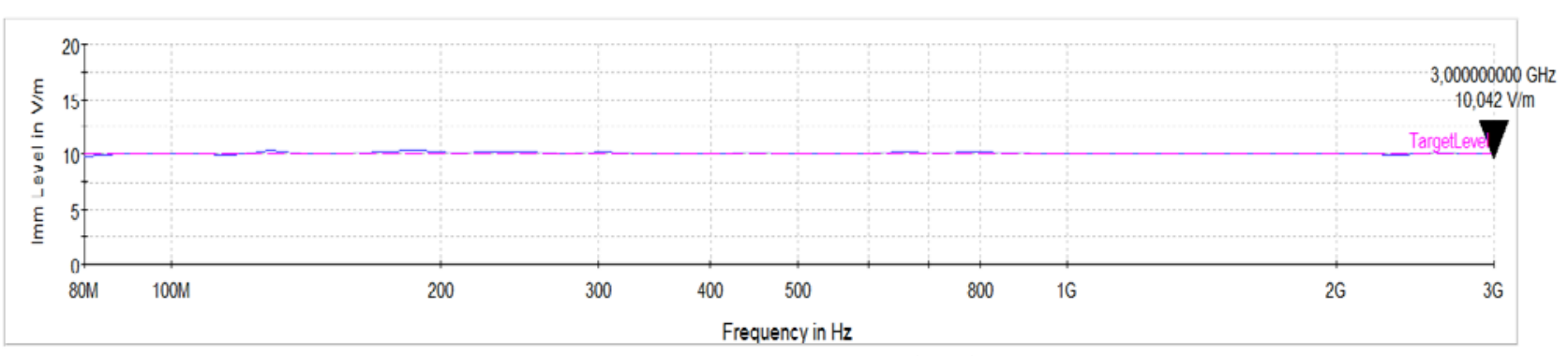

Fig. 5. Test 2 - Immunity level.

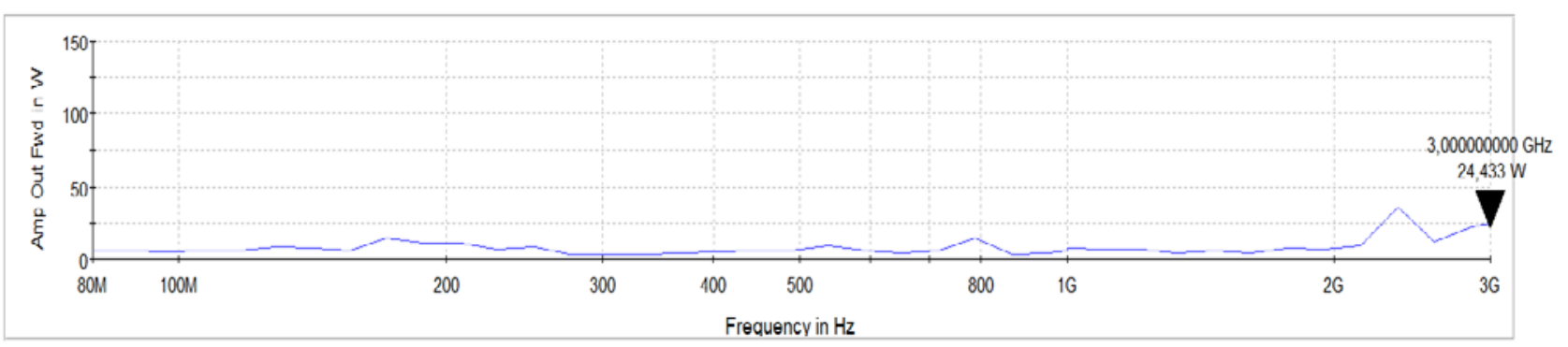

Fig. 6. Test 2 - Amp Out Fwd. 


\section{Conclusion}

The article describes the requirements and the course of the test of electromagnetic susceptibility of electronic devices on radiated radio-frequency electromagnetic field. In our case the EUT was the basic set of the intrusion and hold-up alarm system in two basic states $\mathrm{ON}$ state and state of alarm. For this type of equipment it is necessary to comply not only the basic standard IEC 61000-4-3, but also the next specialized standard EN $50130-4$.

According to standard IEC 61000-4-3 the equipment under test are exposed to test electromagnetic fields with an intensity from $1 \mathrm{~V} / \mathrm{m}$ to $30 \mathrm{~V} / \mathrm{m}$, the most often in the frequency range from $80 \mathrm{MHz}$ to $2 \mathrm{GHz}$. Standard EN 50130-4 recommends testing EMS frequencies up to 2.7 GHz.

During our selected measurements I\&HAS test set was exposed to radio-frequency fields at an intensity of $10 \mathrm{~V} / \mathrm{m}$ and in the frequency range from $80 \mathrm{MHz}$ to 3 GHz. During the immunity test when EUT was in the ON state, the status of the EUT has changed after exceeding the $1.988 \mathrm{GHz}$ frequency - the system detected an error and proclaimed an alarm (the siren was activated). At the end of the test, it was necessary to turn off the alarm manually, but the system remained fully functional. During the immunity test when EUT was in the alarm state, no change was observed.

\section{Acknowledgment}

This work was supported by the Ministry of Education, Youth and Sports of the Czech Republic within the framework of the National Sustainable Development Program no. LO1303 (MSMT-7778/2014) as well as the European Regional Development Fund under the CEBIA-Tech project no. CZ $.1 .05 / 2.1 .00 / 03.0089$ was supported by research project VI20172019054 "Analysis of software module for real-time immunity assessment in terms of convergent security" supported by the Ministry of Interior of the Czech Republic in 2017-2019 and the Internal Grant Agency of Tomas Bata University within the project no. IGA / CebiaTech / 2017/006

\section{References}

1. J. Valouch: Technical requirements for Electromagnetic Compatibility of Alarm Systems. In: International Journal of Circuits, Systems and Signal Processing. Volume 9. USA, Oregon: North Atlantic University Union (2015). p. $186-191$. ISSN: 1998-4464. p. 6.

2. J. Svacina: Electromagnetic compatibility: principles and notes. Issue No. 1. Brno: University of Technology (2001), 156 p. ISBN 8021418737. (in Czech).

3. H. Urbancokova, J. Valouch and M. Adamek: Testing of an Intrusion and Hold-up Systems for Electromagnetic Susceptibility - EFT/B. In: International Journal of Circuits, Systems and Signal Processing. Volume 9. USA, Oregon: North Atlantic University Union (2015). p. 40-46. ISSN: 1998-4464. 7 p.
4. IEC 61000-4-3: Electromagnetic compatibility (EMC) - Part 4-3: Testing and measurement techniques - Radiated, radio-frequency, electromagnetic field immunity test. International Electrotechnical Commission (2006).

5. T. Rybak and M. Steffka: Automotive electromagnetic compatibility (EMC), Boston: Kluwer Academic Publishers (2004), ISBN: 1-40207713-0.

6. H. Ott: Electromagnetic Compatibility. USA, Hoboken: WILEY (2009). ISBN 978-0-470-189306. $844 \mathrm{p}$.

7. EN 50130-4: Alarm systems. Electromagnetic compatibility. Product family standard: Immunity requirements for components of fire, intruder, hold up, CCTV, access control and social alarm systems. European Committee for Electrotechnical Standardization (2011).

8. H. Urbancokova, S. Kovar, J. Valouch and M. Adamek: Electromagnetic Interference of Components of Intrusion and Hold-up Alarm Systems. In: Automation Control Theory Perspectives in Intelligent Systems, Advances in Intelligent Systems and Computing 466 Proceedings of the 5th Computer Science On-line Conference 2016 (CSOC2016) (2016). pp. 443-452 . DOI: 10.1007/978-3-319-33389-2 42. ISBN: 978-3319-33387-8 (Print) 978-3-319-33389-2 (Online). ISSN: 2194-5357. $10 \mathrm{p}$.

9. S. Kovar and J. Valouch: Electromagnetic susceptibility of IP camera. In: Przeglad Elektrotechniczny. Vol 2016, No 5. Poland, Warszawa (2016). pp. 204-208. DOI:10.15199/48.2016.05.40. ISSN 0033-2097. 5 p.

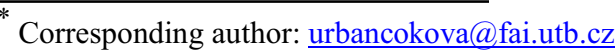

